\title{
DYNAMO ACTION IN ACCRETION DISKS
}

\author{
ULF TORKELSSON \\ Lund Observatory, Box 43, S-221 00 Lund, Sweden
}

\begin{abstract}
Employing the standard theory for thin accretion disks I estimate the relevant parameters for a dynamo in an accretion disk. These estimates could then be compared to the results of numerical simulations. Some preliminary results of such simulations (Torkelsson \& Brandenburg 1992) are presented too.
\end{abstract}

Key words: accretion, accretion disks - dynamo - (MHD) - cataclysmic variables - active galactic nuclei

\section{Introduction}

Using the theory for thin accretion disks (Shakura \& Sunyaev 1973) it is possible to estimate several of the relevant parameters for a dynamo. These estimates are primarily based on the $\alpha$-description of viscous friction. I assume that $\alpha=0.1$ and the magnetic Prandtl number is of order unity. In Tab. $1 M$ is the mass of the compact object, $R_{\text {disk }}$ the radial coordinate for a point in the disk, and $\dot{M}$ the accretion rate. The dynamo numbers are calculated according to $C_{\alpha}=\frac{\alpha_{0} R_{\text {disk }}}{\eta_{\text {disk }}}$ and $C_{\Omega}=\frac{\Omega_{0} R_{\text {disk }}^{2}}{\eta_{\text {disk }}}$, where $\alpha_{0}$ is a typical velocity for the turbulent $\alpha$-effect, $\Omega_{0}$ the angular velocity, and $\eta_{\text {disk }}$ the turbulent magnetic diffusivity in the disk. The given time scales are the Keplerian time scale, $t_{\mathrm{Kepl}}$, and the magnetic diffusivity time scale, $t_{\text {diff }}$. Note that in the numerical calculations I use the diffusivity of the corona instead, which is assumed to be 20 times larger. The magnetic field, $B_{\text {press }}$ is estimated by equilibrating the gas and magnetic pressure. Finally I give the temperature $T$ of the disk. The low dynamo numbers for the AGN is due to the choice of a high accretion rate and low mass for the black hole.

\section{Numerical simulations}

We have undertaken numerical simulations of a disk dynamo by solving the dynamo equation

$$
\frac{\partial \mathbf{B}}{\partial t}=\boldsymbol{\nabla} \times(\mathbf{V} \times \mathbf{B}+\alpha \mathbf{B})-\boldsymbol{\nabla} \times\left(\eta_{\mathrm{t}} \boldsymbol{\nabla} \times \mathbf{B}\right),
$$

(Torkelsson \& Brandenburg 1992). It is solved with a time-stepping method on a 2-dimensional grid in the $r \theta$-plane, where $r$ and $\theta$ are spherical coordinates ranging from 0 to 1 , and 0 to $\frac{\pi}{2}$ or $\pi$, respectively (Brandenburg et al. 1989). We assume Keplerian rotation in the disk except in the innermost $25 \%$ where it turns over into rigid rotation. The magnetic diffusivity is small, 0.05 , and constant inside the disk, and 1 outside the disk, to simulate a surrounding vacuum. Finally the $\alpha$-effect is proportional to the angular velocity $\Omega$ and the vertical coordinate $z$.

An example of a simulation is presented in Fig. 1. If one decreases the thickness of this disk, it will be easier to excite a steady S0 mode than the oscillating A0 mode, which is in agreement with Stepinski \& Levy (1990). 
TABLE I

Magnetic fields and time scales in accretion disks

\begin{tabular}{lrrrr}
\hline Object & White dwarf & Neutron star & Stellar black hole & Black hole in AGN \\
\hline$M\left(M_{\odot}\right)$ & 1 & 1 & 10 & $10^{7}$ \\
$R_{\text {disk }}(\mathrm{m})$ & $10^{7}$ & $310^{6}$ & $10^{6}$ & $10^{12}$ \\
$\dot{M}\left(M_{\odot} \mathrm{yr}^{-1}\right)$ & $810^{-9}$ & $10^{-9}$ & $210^{-9}$ & 1 \\
$C_{\alpha}$ & 70 & 100 & 500 & 2 \\
$C_{\Omega}$ & 5000 & 20000 & 200000 & 3 \\
$t_{\text {Kepl }}(\mathrm{s})$ & 20 & 0.02 & 0.006 & 6000 \\
$t_{\text {diff }}(\mathrm{s})$ & 10000 & 60 & 200 & 3000 \\
$B_{\text {press }}(\mathrm{T})$ & 80 & 10000 & 20000 & 0.03 \\
$T(\mathrm{eV})$ & 70 & 2000 & 3000 & 60
\end{tabular}

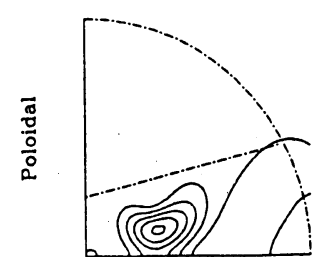

$\min -0.0006 \max 0.0036$

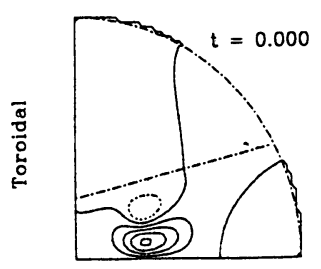

$\min -0.7 \max 1.7$

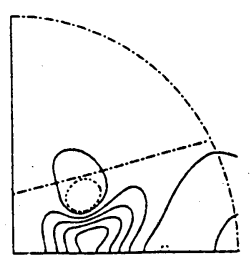

$\min -0.0009 \max 0.0029$

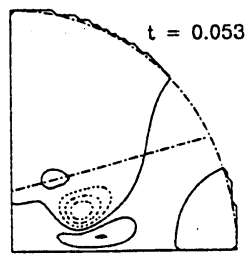

$\min -1.2 \max 0.6$

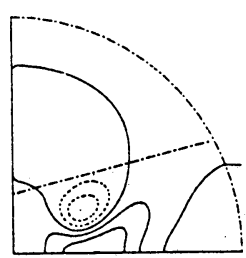

$\min -0.0021 \max =0.0021$

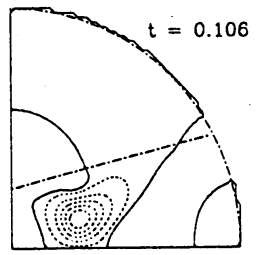

$\min -1.7 \max 0.2$

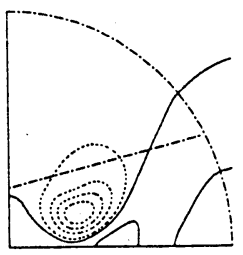

$\min -0.0032 \max 0.001 \mathrm{i}$

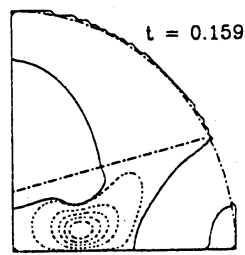

$\min -2.1 \max 0.3$

Fig. 1. For a disk with thickness 0.25 at the rotational axis and thickening outwards with a slope of 0.25 , the most easily excited mode is an oscillating $\mathrm{A} 0$ mode with $C_{\alpha} C_{\Omega}=43.2$ and angular frequency of 14.7 in units of the inverse of the magnetic diffusivity time outside the disk. This is in agreement with Stepinski \& Levy (1988). The upper row of the figure shows the poloidal field and the lower one the toroidal field, solid lines are for positive values and broken lines for negative. $t=0$ is chosen arbitrarily.

\section{Acknowledgements}

The numerical calculations are being carried out on the Cray X-MP/416 at the National Supercomputer Center, Linköping, Sweden.

\section{References}

Brandenburg, A., et al: 1989, $A \& A$ 213, 411

Shakura, N. I. and Sunyaev, R. A.: 1973, $A \& A$ 24, 337

Stepinski, T. F. and Levy, E. H.: 1988, $A p J$ 331, 416

Stepinski, T. F. and Levy, E. H.: 1990, ApJ 362, 318

Torkelsson, U. and Brandenburg, A.: 1992, $A \mathcal{G} A$, in preparation 